\title{
Should we target "intermediate expression" of HER2 in older estrogen receptor positive patients?
}

\author{
Yoshihisa Tokumaru ${ }^{1,2}$, Lan Le ${ }^{1,3}$, Mariko Asaoka $^{4}$, Manabu Futamura ${ }^{2}$, Takashi Ishikawa ${ }^{4}$, \\ Kazuhiro Yoshida ${ }^{2}$, Kazuaki Takabe ${ }^{1,4,5,6,7}$
}

${ }^{1}$ Breast Surgery, Department of Surgical Oncology, Roswell Park Comprehensive Cancer Center, Buffalo, NY, USA; ${ }^{2}$ Department of Surgical Oncology, Graduate School of Medicine, Gifu University, Gifu, Japan; ${ }^{3}$ Department of Surgery, University at Buffalo Jacobs School of Medicine and Biomedical Sciences, The State University of New York, Buffalo, NY, USA; ${ }^{4}$ Department of Breast Surgery and Oncology, Tokyo Medical University, Tokyo, Japan; ${ }^{5}$ Department of Surgery, Yokohama City University, Yokohama, Japan; ${ }^{6}$ Department of Surgery, Niigata University Graduate School of Medical and Dental Sciences, Niigata, Japan; ${ }^{7}$ Department of Breast Surgery, Fukushima Medical University School of Medicine, Fukushima, Japan Correspondence to: Dr. Kazuaki Takabe, MD, PhD, FACS. Breast Surgery, Department of Surgical Oncology Roswell Park Comprehensive Cancer Center, Elm \& Carlton Streets, Buffalo, NY 14263, USA. Email: kazuaki.takabe@roswellpark.org.

Provenance and Peer Review: This article was commissioned by the editorial office, Translational Cancer Research. The article did not undergo external peer review.

Comment on: Kim MH, Kim GM, Kim JH, et al. Intermediate HER2 expression is associated with poor prognosis in estrogen receptor-positive breast cancer patients aged 55 years and older. Breast Cancer Res Treat 2020;179:687-97.

Submitted Jun 01, 2020. Accepted for publication Jun 29, 2020.

doi: $10.21037 /$ tcr-20-2231

View this article at: http://dx.doi.org/10.21037/tcr-20-2231

Breast cancer is a heterogeneous disease, which is phenotypically diverse in response to treatment as well as clinical outcomes (1). Charles Perou established the four molecular subtypes using gene expression patterns, which is now the foundation of how we categorize breast cancers (2). Breast cancer subtypes are simplified in clinical practice where it is categorized into three major subtypes based on three receptor expressions; estrogen receptor (ER)-positive, human epidermal growth factor receptor 2 (HER2)-positive and triple negative where ER, HER2, and progesterone receptor $(\mathrm{PR})$ are negative. Determination of the subtype by receptors is essential in the clinical setting because it guides the selection of treatments of the patient; anti-estrogen hormonal therapy for ER-positive, antiHER2 targeted therapy for HER2-positive and cytotoxic chemotherapy for triple negative subtype (3). There are four subtypes in the original Perou's classification; luminal A, luminal B, HER2-enriched, and basal-like subtype, where ER-positives are divided into Ki-67 expression low luminal A and high luminal B. In general, ER-positive breast cancer patients demonstrate better prognosis compared with other subtypes. However, it is known that these tumors do recur in the long run (4). The 20-year recurrence rate for ER- positive breast cancer ranges from $10 \%$ to $41 \%$ (5).

In the current clinical practice, HER2-positive is defined by high positivity (3+) in immunohistochemistry (IHC) or intermediate positivity (2+) in IHC and fluorescence in situ hybridization (FISH)-positive (6). Intermediate expression of HER2 is commonly defined as intermediate positivity (2+) in IHC and FISH-negative, whereas HER2-negative is defined as either negative or low positivity (1+) in IHC and FISH-negative (6). Compared with HER2-negative, breast cancer with an intermediate expression of HER2 has more aggressive characteristics as well as worse prognosis. This is in agreement with the fact that the HER2 signal pathway is associated with aggressive features and risks of recurrence $(7,8)$. Furthermore, these results raise the notion that HER2 expression may not be simply positive or negative but a continuum, and HER2-intermediate expression breast cancer may still respond to anti-HER2 therapy even if their expression is moderate.

To date, the prognostic and predictive value of HER2intermediate expression is very limited. Ignatov et al. reported that when compared with 4,073 HER2-negative tumors (defined by IHC negative or 1+), 822 HER2 moderate tumors (defined as IHC $2+$ and FISH-positive) 
demonstrated aggressive features and worse prognosis (9). Also, Rossi et al., reported that when compared to the patients with IHC negative or $1+$, the patients with IHC $2+$ and FISH-negative demonstrated worse disease-free survival (DFS) (10). On the other hand, the addition of trastuzumab (anti-HER2 targeted therapy) to adjuvant chemotherapy (docetaxel plus cyclophosphamide or doxorubicin and cyclophosphamide followed by weekly paclitaxel) did not improve the survival of the patients with non-HER2 overexpressing patients (IHC $1+$ or $2+$ with FISH-negative) in the randomized and multi-center clinical trial, NSABP B-47 (NCT01275677) (11). They concluded that the addition of trastuzumab to adjuvant chemotherapy did not improve, all the survivals analyzed, such as DFS, recurrence-free survival, and overall survival. Since this study included the patients with IHC 1+ to the study group, this result is against the notion to treat HER2-intermediate expression group with anti-HER2 therapy. Therefore, it has been an interest in the field to explore the benefit of antiHER2 targeted therapy with chemotherapy against HER2intermediate expression group, which is defined as IHC 2+ and FISH-negative.

In the study by Kim et al., they prospectively analyzed the early breast cancer patients and metastatic breast cancer patients of ER positive subtype (12). They categorized those patients into three groups utilizing HER2 IHC status. They defined HER2-negative as IHC 0-1, HER2intermediate as IHC 2+, and FISH-negative. A total of 2,657 patients were enrolled to analyze in order to elucidate the prognostic impact of intermediate HER2 expression in early breast cancer or metastatic breast cancer of ERpositive subtype. Among those, there were 654 early breast cancer patients and 166 metastatic breast cancer patients which were defined as the HER2-intermediate group. On the other hand, the HER2-negative group consisted of 2,003 early breast cancer and 369 metastatic breast cancer patients in the HER2-intermediate group. The survival analysis revealed that when compared with the HER2 negative group, the HER2-intermediate group demonstrated worse recurrence-free survival (RFS) $(\mathrm{P}=0.044)$. Interestingly, among those, only the early breast cancer patients with $\geq 55$ years predicted to have poorer recurrence free survival [hazard ratio (HR) 1.95; $\mathrm{P}=0.042$ ] whereas $<55$ years did not. HER2-intermediate group demonstrated worse survival in metastatic breast cancer patients as well. The HER2intermediate group demonstrated worse overall survival than the HER2 negative group patients with $\geq 55$ years $(\mathrm{P}=0.037)$, whereas there was no significant difference between those groups in patients with $<55$ years $(\mathrm{P}=0.163)$.

The worse prognosis observed in the HER2-intermediate group compared to the HER2-negative group is consistent with the previous reports $(9,10)$. What was unique in Kim et al.'s work is its focus on the age, $\geq 55$ years, which was different from other studies and had a very intriguing finding. Generally, younger age is often reported to be a prognostic factor of breast cancer patients. Numerous reports have demonstrated worse prognosis with younger patients than older patients $(1,13,14)$. In many studies, younger patients are defined as age under 40 years old (14). The worse prognosis with younger patients is thought to associate with the fact that younger breast cancer patients tend to possess more aggressive biological characteristics $(1,13)$. Recent studies elucidated that the worse prognosis of younger patients depends on molecular subtypes. Partridge et al. (1) reported that younger age (age $\leq 40$ years old) increased the risk of disease specific death in luminal subtypes alone. Concordance with this report, Lian et al. (14) reported that younger age (age $\leq 40$ years old) was the risk factor for inferior survival in luminal A and luminal B (HER2negative) subtypes only, whereas luminal B (HER2-positive), HER2 overexpression, and triple negative subtypes were not. Furthermore, the result of the TAILORx trial, in which the risk of recurrence score was calculated based on the expression of 21 genes, the benefit of chemotherapy was demonstrated in the patients under 50 years of age in recurrence score of 16-25 population (15). To this end, it was very surprising to find that the patients with over 55 years of age were the risk factor of poor prognosis.

Our group in collaboration with multiple institutions has recently published that HER2 positivity of primary breast cancer was the risk factor of recurrence after neoadjuvant chemotherapy and associate with worse survival (8). Among the subtypes analyzed in this study, HER2, luminal-HER2, luminal, and triple negative, HER2 subtype demonstrated a significantly higher risk of recurrence compared with the other three subtypes (hazard ratio $=2.6, \mathrm{P}=0.02$ ). Also, when compared with HER2 negative breast cancer, HER2 positive breast cancer was associated with recurrence (hazard ratio $=2.5, \mathrm{P}=0.04)$. Interestingly, we also found that HER2 positive subtype demonstrated the worst DFS among the four subtypes analyzed $(\mathrm{P}=0.048)$. Furthermore, HER2positive breast cancer patients demonstrated worse DFS compared with HER2-negative breast cancer patients.

Given that the estrogen signaling and HER2 signaling are the two major signaling pathways that drive breast cancer growth, the activation of HER2 has been postulated 
to be associated with tumor recurrence in pre-clinical basic research study as well. The crosstalk between the ER and HER2 pathways have been reported, suggesting that HER2 pathway activity may promote aggressiveness and endocrine resistance in ER-positive breast cancer, even in cases of tumors without HER2 amplification (16). To this end, we cannot help but speculate that HER2 expression levels may be a candidate to distinguish the long-term recurrence of ER-positive breast cancer.

In conclusion, sub-categorizing ER-positive breast cancer by age and HER2 status may help us predict the patient outcome more precisely.

\section{Acknowledgments}

Funding: This work was supported by NIH grant R01CA160688 and Susan G. Komen Foundation grant CCR17481211 to KT, and NCI cancer center support grant P30-CA016056 to Roswell Park Comprehensive Cancer Center (RPCCC).

\section{Footnote}

Conflicts of Interest: All authors have completed the ICMJE uniform disclosure form (available at http:// dx.doi.org/10.21037/tcr-20-2231). KY reports grants and personal fees from Taiho Pharm, Asahi Kasei Pharma, Chugai Pharm., Covidien Japan, Daiichi Sankyo, Eisai, Eli Lilly Japan, Johnson \& Johnson, MerkSerono, MSD, Nippon Kayaku, Novartis, Ono Pharm., Otsuka Pharm., Sanofi, Takeda Pharm., Tsumura, Yakult Honsha; grants from Abbott, Abbvie, Astellas, Biogen Japan, Celgene, GlaxoSmithKline, KCI, Koninklijke Philips, Kyowa Kirin, Meiji Seika Pharma, Toray Medical; personal fees from AstraZeneka, Bristol-Myers Squibb, Denka, EA Pharma, Olympus, Pfizer, Sanwa Kagaku Kenkyusho, SBI Pharma, Teijin Phamra, TERUMO, outside the submitted work. KT reports grants from National Institute of Health (USA), grants from National Institute of Health (USA), during the conduct of the study. The other authors have no conflicts of interest to declare.

Ethical Statement: The authors are accountable for all aspects of the work in ensuring that questions related to the accuracy or integrity of any part of the work are appropriately investigated and resolved.

Open Access Statement: This is an Open Access article distributed in accordance with the Creative Commons Attribution-NonCommercial-NoDerivs 4.0 International License (CC BY-NC-ND 4.0), which permits the noncommercial replication and distribution of the article with the strict proviso that no changes or edits are made and the original work is properly cited (including links to both the formal publication through the relevant DOI and the license). See: https://creativecommons.org/licenses/by-nc-nd/4.0/.

\section{References}

1. Partridge AH, Hughes ME, Warner ET, et al. SubtypeDependent Relationship Between Young Age at Diagnosis and Breast Cancer Survival. J Clin Oncol 2016;34:3308-14.

2. Perou CM, Sørlie T, Eisen MB, et al. Molecular portraits of human breast tumours. Nature 2000;406:747-52.

3. Tsuchida J, Nagahashi M, Takabe K, et al. Clinical Impact of Sphingosine-1-Phosphate in Breast Cancer. Mediators Inflamm 2017;2017:2076239.

4. Takeshita T, Yan L, Asaoka M, et al. Late recurrence of breast cancer is associated with pro-cancerous immune microenvironment in the primary tumor. Sci Rep 2019;9:16942.

5. Tokumaru Y, Joyce D, Takabe K. Current status and limitations of immunotherapy for breast cancer. Surgery 2020;167:628-30.

6. Wolff AC, Hammond MEH, Allison KH, et al. Human Epidermal Growth Factor Receptor 2 Testing in Breast Cancer: American Society of Clinical Oncology/College of American Pathologists Clinical Practice Guideline Focused Update. J Clin Oncol 2018;36:2105-22.

7. Pietras RJ, Márquez-Garbán DC. Membrane-associated estrogen receptor signaling pathways in human cancers. Clin Cancer Res 2007;13:4672-6.

8. Asaoka M, Narui K, Suganuma N, et al. Clinical and pathological predictors of recurrence in breast cancer patients achieving pathological complete response to neoadjuvant chemotherapy. Eur J Surg Oncol 2019;45:2289-94.

9. Ignatov T, Eggemann H, Burger E, et al. Moderate level of HER2 expression and its prognostic significance in breast cancer with intermediate grade. Breast Cancer Res Treat 2015;151:357-64.

10. Rossi V, Sarotto I, Maggiorotto F, et al. Moderate immunohistochemical expression of HER-2 (2+) without HER-2 gene amplification is a negative prognostic factor in early breast cancer. Oncologist 2012;17:1418-25. 
11. Fehrenbacher L, Cecchini RS, Geyer CE Jr, et al. NSABP B-47/NRG Oncology Phase III Randomized Trial Comparing Adjuvant Chemotherapy With or Without Trastuzumab in High-Risk Invasive Breast Cancer Negative for HER2 by FISH and With IHC 1+ or 2. J Clin Oncol 2020;38:444-53.

12. Kim MH, Kim GM, Kim JH, et al. Intermediate HER2 expression is associated with poor prognosis in estrogen receptor-positive breast cancer patients aged 55 years and older. Breast Cancer Res Treat 2020;179:687-97.

13. Anders CK, Johnson R, Litton J, et al. Breast cancer before age 40 years. Semin Oncol 2009;36:237-49.

14. Lian W, Fu F, Lin Y, et al. The Impact of Young Age for Prognosis by Subtype in Women with Early Breast Cancer. Sci Rep 2017;7:11625.

15. Sparano JA, Gray RJ, Makower DF, et al. Adjuvant Chemotherapy Guided by a 21-Gene Expression Assay in Breast Cancer. N Engl J Med 2018;379:111-21.

16. Osborne CK, Schiff R. Growth factor receptor crosstalk with estrogen receptor as a mechanism for tamoxifen resistance in breast cancer. Breast 2003;12:362-7.
Cite this article as: Tokumaru Y, Le L, Asaoka M, Futamura M, Ishikawa T, Yoshida K, Takabe K. Should we target "intermediate expression" of HER2 in older estrogen receptor positive patients? Transl Cancer Res 2020;9(7):4056-4059. doi: $10.21037 /$ tcr-20-2231 